\title{
Profiles and Clinical Characteristics of Newly Diagnosed Glaucoma in Urban Korea: A Multicenter Study
}

\author{
Chul Hong', Seung Woo Hong ${ }^{2}$, Chan Kee Park ${ }^{3}$, Kyung Rim Sung ${ }^{4}$, Chang-sik Kim ; on behalf of Korean Glaucoma \\ Society \\ ${ }^{1}$ Glaucoma Service, Dr. Hong's Eye Clinic, Seoul, Korea \\ ${ }^{2}$ Department of Ophthalmology, Bucheon St. Mary's Hospital, Bucheon, Korea \\ ${ }^{3}$ Department of Ophthalmology, Seoul St. Mary's Hospital, Seoul, Korea \\ ${ }^{4}$ Department of Ophthalmology, Asan Medical Center, Seoul, Korea \\ ${ }^{5}$ Department of Ophthalmology, Chungnam National University Hospital, Daejeon, Korea
}

\begin{abstract}
Purpose: To explore the clinical characteristics and profiles of newly diagnosed glaucoma subtypes in urban Korea.
Methods: All newly diagnosed glaucoma patients enrolled in the participating ophthalmology outpatient clinics were included. A review of medical history including family history of glaucoma was conducted. The patients underwent complete ophthalmologic examinations including visual field test. The diagnosis of glaucoma was based on the International Society of Geographical and Epidemiological Ophthalmology criteria developed by glaucoma specialists.

Results: A total of 198,671 patients visited the participating ophthalmology outpatient clinics during the study period (from January 1, 2001 to June 30, 2016), of which 5,530 (2.8\%) were diagnosed with glaucoma. The mean age of the newly diagnosed glaucoma patients was $52.0 \pm 17.3$ years (range, 6 to 89 ) and 2,830 patients were male (51.2\%). The mean untreated intraocular pressure and vertical cup-to-disc ratio of the optic nerve head of newly diagnosed glaucoma eyes were $22.1 \pm 10.6$ $\mathrm{mmHg}$ and $0.66 \pm 0.22$, respectively. The most frequently observed subtypes of glaucoma were: normal tension glaucoma (33.0\%) primary open-angle glaucoma (28.4\%), ocular hypertension (11.1\%), chronic angle-closure glaucoma (6.8\%), neovascular glaucoma (5.2\%), glaucoma associated with inflammation (3.8\%), acute angle-closure glaucoma (3.3\%), and glaucoma associated with aphakia or pseudophakia (2.2\%).
\end{abstract}

Conclusions: Normal tension glaucoma was the most frequently observed glaucoma subtype in urban ophthalmology outpatient clinics in Korea.

Key Words: Epidemiology, Glaucoma, Low tension glaucoma, Open-angle glaucoma

Glaucoma is one of the leading causes of blindness worldwide [1-3]. Among the subtypes of glaucoma, primary

Received: March 19, 2020 Final revision: May 12, 2020

Accepted: May 15, 2020

Correspondence Author: Chang-sik Kim, MD, PhD. Department of Ophthalmology, Chungnam National University Hospital, 282 Munhwa-ro, Jung-gu, Daejeon 35015, Korea. Tel: 82-42-280-7604, Fax: 82-42-2553745, E-mail:kcs61@cnu.ac.kr open-angle glaucoma (POAG) was the most common type in many population-based studies [4-11]. However, the prevalence of glaucoma and its proportion of subtypes vary by nationality [12-15]. Also, in some studies, the prevalence of subtypes shows regional difference within the same country [16,17].

According to the population-based Namil study, the prevalence of POAG in Korea was 3.59\% [18]. When 21 
mmHg was used as the cut-off value, the prevalence of POAG with intraocular pressure (IOP) $\leq 21 \mathrm{mmHg}$ was $2.81 \%$ and $>21 \mathrm{mmHg}$ was $0.78 \%$. The Namil study was conducted in rural areas, and the prevalence of glaucoma subtypes varies between rural and urban areas. Most Koreans live in cities $(81.5 \%$ of total population, from Bureau of Korean Statistics). Therefore, knowledge of the subtypes prevalent in the urban areas is important. However, the investigation of the prevalence of glaucoma in urban Korean society proved to be difficult because of the low compliance and participation rates. In fact, several attempts have already failed.

Thus, in this multicenter study, we investigated the profile of newly diagnosed glaucoma subtypes in an outpatient ophthalmology clinic located in an urban area. With the enrollment of 20 major clinics located in urban area, we intended to review the clinical characteristics of main glaucoma subtypes. Most of the university-based ophthalmology outpatient clinics and 41 members of Korean Glaucoma Society participated in this study allowing us to generalize the results.

\section{Materials and Methods}

All participating hospitals and ophthalmology outpatient clinics were located in major cities in Korea. From January 1, 2001 to June 30, 2016, all newly diagnosed glaucoma patients visiting those clinics were enrolled. The study protocol was approved by the institutional review board of each university hospital, and subsequent investigations followed the tenets of the Declaration of Helsinki. Informed consent was waived due to retrospective nature of the study design. The newly diagnosed glaucoma patients were interviewed for a medical history that included a family history of glaucoma. Eye examinations were performed including refractive error measurement, standard visual acuity testing with determination of best refractive correction, slit-lamp examination including Van Herick measurement of anterior chamber depth, IOP measurement with Goldmann applanation tonometry, fundus examination including binocular optic disc evaluation, a gonioscopic examination followed by visual field testing (Humphrey Field Analyzer; Carl Zeiss Meditec, Dublin, CA, USA), and structural evaluation using Stratus optical coherence tomography (Carl Zeiss Meditec) and/or scanning laser polarimetry
(GDx Vcc, Carl Zeiss Meditec). Newly diagnosed glaucoma patients were classified according to the International Society of Geographical \& Epidemiological Ophthalmology criteria. These classifications are divided into $26 \mathrm{sub}-$ types: ocular hypertension (OHT); POAG; normal tension glaucoma (NTG); acute angle-closure glaucoma (AACG); subacute ACG; chronic angle-closure glaucoma (CACG); plateau iris syndrome; combined-mechanism glaucoma; congenital glaucoma; glaucoma associated with developmental anomaly (Axenfeld-Rieger syndrome, Peter anomaly, aniridia, and Sturge-Weber syndrome); steroid-induced glaucoma; glaucoma associated with corneal endothelial disorders (iridocorneal endothelial syndrome and Fuch's endothelial dystrophy); pigmentary glaucoma; exfoliation (pseudoexfoliation) syndrome; glaucoma associated with cataract; glaucoma associated with lens dislocation; neovascular glaucoma (NVG); glaucoma associated with retinal detachment or vitreoretinal abnormality; glaucoma associated with inflammation (Posner-Schlossman syndrome, uveitis, keratitis, episcleritis, and scleritis); angle-recession glaucoma; glaucoma associated with hemorrhage; ciliary block (malignant) glaucoma; glaucoma in aphakia or pseudophakia; glaucoma associated with corneal surgery; glaucoma associated with vitreoretinal surgery; and others. If both eyes were included, the eye most severely affected in terms of visual field mean deviation was used for classification.

\section{Statistical analysis}

Age, sex, involved eye, untreated IOP, family history of glaucoma, presence of diabetes or systemic hypertension, and type of newly diagnosed glaucoma were recorded. All data for newly diagnosed glaucoma patients were collected and analyzed by one designated author (HC). Overlapping patients were excluded. Statistical analysis was performed using PASW Statistics ver. 18.0 (SPSS Inc., Chicago, IL, USA). A $p$-value $<0.05$ level was considered as significant for all tests.

\section{Results}

During the study period, a total of 198,671 patients visited the participating ophthalmology outpatient clinics. Among them, 5,530 (2.8\%) were diagnosed with glaucoma 
Table 1. Demographics and medical history of newly diagnosed glaucoma patients

\begin{tabular}{|c|c|c|c|c|c|c|}
\hline Diagnosis & No. of patients & Mean age (yr) & $\begin{array}{l}\text { Sex ratio } \\
(\%)\end{array}$ & $\begin{array}{c}\text { Family } \\
\text { history (\%) }\end{array}$ & $\begin{array}{l}\text { Diabetes } \\
(\%)\end{array}$ & $\begin{array}{l}\text { Hypertension } \\
(\%)\end{array}$ \\
\hline Ocular hypertension & $612(11.1 \%)$ & $42.92 \pm 16.51$ & 47.06 & 6.37 & 8.50 & 13.73 \\
\hline Primary open-angle glaucoma & $1,572(28.4 \%)$ & $52.74 \pm 17.25$ & 42.23 & 5.66 & 11.90 & 17.62 \\
\hline Normal-tension glaucoma & $1,825(33.0 \%)$ & $51.38 \pm 15.97$ & 51.45 & 6.19 & 9.32 & 18.79 \\
\hline Acute angle-closure glaucoma & $186(3.4 \%)$ & $64.28 \pm 10.18$ & 75.81 & 1.65 & 9.14 & 23.11 \\
\hline Subacute angle-closure glaucoma & $37(0.7 \%)$ & $65.00 \pm 11.38$ & 75.68 & 5.41 & 10.81 & 37.84 \\
\hline Chronic angle-closure glaucoma & $378(6.8 \%)$ & $64.88 \pm 10.37$ & 68.71 & 4.69 & 8.66 & 23.83 \\
\hline Plateau iris syndrome & $2(0 \%)$ & $63.00 \pm 4.24$ & 50.00 & 0.00 & 0.00 & 0.00 \\
\hline Angle-recession glaucoma & $23(0.4 \%)$ & $39.13 \pm 15.33$ & 13.04 & 8.70 & 4.35 & 8.70 \\
\hline Glaucoma associated with cataract & $31(0.6 \%)$ & $66.61 \pm 18.40$ & 54.84 & 3.23 & 6.45 & 16.13 \\
\hline Lens dislocation & $10(0.2 \%)$ & $55.10 \pm 16.50$ & 10.00 & 0.00 & 20.00 & 10.00 \\
\hline Pseudoexfoliation syndrome & $41(0.7 \%)$ & $66.90 \pm 12.67$ & 39.02 & 4.88 & 4.88 & 29.27 \\
\hline Steroid-induced glaucoma & $67(1.2 \%)$ & $37.35 \pm 16.20$ & 49.25 & 1.49 & 7.46 & 8.96 \\
\hline Glaucoma associated with hemorrhage & $19(0.3 \%)$ & $45.63 \pm 18.01$ & 26.32 & 0.00 & 10.53 & 15.79 \\
\hline Combined-mechanism glaucoma & $13(0.2 \%)$ & $57.33 \pm 21.19$ & 46.13 & 0.00 & 7.69 & 23.08 \\
\hline Congenital glaucoma & $37(0.7 \%)$ & $12.22 \pm 11.88$ & 37.84 & 8.11 & 0.00 & 0.00 \\
\hline $\begin{array}{l}\text { Glaucoma associated with developmental } \\
\text { anomaly }\end{array}$ & $3(0.1 \%)$ & $18.00 \pm 15.88$ & 0.00 & 0.00 & 0.00 & 0.00 \\
\hline $\begin{array}{l}\text { Glaucoma associated with corneal endothelial } \\
\text { disorder }\end{array}$ & $13(0.2 \%)$ & $42.85 \pm 11.55$ & 61.54 & 0.00 & 15.38 & 15.38 \\
\hline Pigmentary glaucoma & $9(0.2 \%)$ & $47.33 \pm 12.30$ & 11.11 & 0.00 & 11.11 & 22.22 \\
\hline Neovascular glaucoma & $289(5.2 \%)$ & $56.90 \pm 15.72$ & 41.18 & 2.08 & 47.40 & 31.49 \\
\hline $\begin{array}{l}\text { Glaucoma associated with retinal detachment or } \\
\text { vitreous abnormality }\end{array}$ & $10(0.2 \%)$ & $49.90 \pm 21.64$ & 30.00 & 0.00 & 10.00 & 30.00 \\
\hline Glaucoma associated with inflammation & $211(3.8 \%)$ & $48.50 \pm 15.73$ & 41.71 & 1.42 & 5.21 & 9.95 \\
\hline Ciliary black glaucoma & $11(0.2 \%)$ & $61.27 \pm 10.52$ & 36.36 & 0.00 & 0.00 & 9.09 \\
\hline Glaucoma in aphakia or pseudophakia & $121(2.2 \%)$ & $59.18 \pm 13.90$ & 28.93 & 3.31 & 14.05 & 19.01 \\
\hline Glaucoma associated with corneal surgery & $9(0.2 \%)$ & $51.22 \pm 15.06$ & 33.33 & 0.00 & 33.33 & 11.11 \\
\hline Glaucoma associated with vitreoretinal surgery & $23(0.4 \%)$ & $49.39 \pm 18.00$ & 43.48 & 0.00 & 30.43 & 17.39 \\
\hline Others & $81(1.5 \%)$ & $48.23 \pm 17.00$ & 46.91 & 2.47 & 4.94 & 17.28 \\
\hline Total & $5,530(100 \%)$ & & & & & \\
\hline
\end{tabular}

which belonged to one of the above mentioned International Society of Geographical \& Epidemiological Ophthalmology categories. The mean age of the newly diagnosed glaucoma patients was $52.0 \pm 17.3$ years (range, 6 to 89 ) of which 2,830 were male (51.2\%). The mean IOP and vertical cup-to-disc ratio of the optic disc head in newly diagnosed glaucoma patients were $22.1 \pm 10.6 \mathrm{mmHg}$ and $0.66 \pm 0.22$, respectively. The proportions of the glaucoma subtypes are shown in Table 1 . The most frequently observed glaucoma subtypes were NTG (33.0\%), POAG (28.4\%), OHT (11.1\%), CACG (6.8\%), NVG (5.2\%), glaucoma associated with inflammation (3.8\%), AACG (3.4\%), and glaucoma in apha- 
Table 2. Demographics and medical history of newly diagnosed glaucoma patients

\begin{tabular}{|c|c|c|}
\hline Diagnosis & Mean IOP (mmHg) & Mean vertical cup-to-disc ratio \\
\hline Ocular hypertension & $21.81 \pm 4.78$ & $0.524 \pm 0.210$ \\
\hline Primary open-angle glaucoma & $22.19 \pm 8.17$ & $0.696 \pm 0.226$ \\
\hline Normal-tension glaucoma & $16.31 \pm 3.48$ & $0.691 \pm 0.166$ \\
\hline Acute angle-closure glaucoma & $37.34 \pm 19.41$ & $0.492 \pm 0.261$ \\
\hline Subacute angle-closure glaucoma & $21.81 \pm 8.92$ & $0.578 \pm 0.227$ \\
\hline Chronic angle-closure glaucoma & $24.43 \pm 12.53$ & $0.679 \pm 0.268$ \\
\hline Plateau iris syndrome & $12.50 \pm 2.12$ & $0.500 \pm 0.424$ \\
\hline Angle-recession glaucoma & $31.04 \pm 12.88$ & $0.548 \pm 0.254$ \\
\hline Glaucoma associated with cataract & $37.37 \pm 18.84$ & $0.511 \pm 0.214$ \\
\hline Lens dislocation & $38.11 \pm 13.19$ & $0.483 \pm 0.214$ \\
\hline Pseudoexfoliation syndrome & $21.12 \pm 7.85$ & $0.740 \pm 0.228$ \\
\hline Steroid-induced glaucoma & $25.44 \pm 8.76$ & $0.604 \pm 0.215$ \\
\hline Glaucoma associated with hemorrhage & $33.63 \pm 14.03$ & $0.692 \pm 0.193$ \\
\hline Combined-mechanism glaucoma & $26.58 \pm 8.70$ & $0.741 \pm 0.253$ \\
\hline Congenital glaucoma & $24.36 \pm 9.80$ & $0.718 \pm 0.219$ \\
\hline Glaucoma associated with developmental anomaly & $37.33 \pm 8.02$ & $0.733 \pm 0.306$ \\
\hline Glaucoma associated with corneal endothelial disorder & $27.00 \pm 12.29$ & $0.654 \pm 0.163$ \\
\hline Pigmentary glaucoma & $27.78 \pm 14.53$ & $0.733 \pm 0.233$ \\
\hline Neovascular glaucoma & $34.49 \pm 16.87$ & $0.686 \pm 0.227$ \\
\hline Glaucoma associated with retinal detachment or vitreous abnormality & $24.40 \pm 10.28$ & $0.688 \pm 0.253$ \\
\hline Glaucoma associated with inflammation & $28.66 \pm 12.70$ & $0.571 \pm 0.236$ \\
\hline Ciliary black glaucoma & $41.55 \pm 16.24$ & $0.567 \pm 0.301$ \\
\hline Glaucoma in aphakia or pseudophakia & $26.08 \pm 11.47$ & $0.716 \pm 0.224$ \\
\hline Glaucoma associated with corneal surgery & $29.50 \pm 9.20$ & $0.600 \pm 0.187$ \\
\hline Glaucoma associated with vitreoretinal surgery & $30.09 \pm 14.34$ & $0.693 \pm 0.237$ \\
\hline Others & $20.85 \pm 8.49$ & $0.613 \pm 0.194$ \\
\hline
\end{tabular}

$\mathrm{IOP}=$ intraocular pressure

kia or pseudophakia $(2.2 \%)$.

The demographics and concurrent medical conditions of the newly diagnosed glaucoma patients are also shown in Table 1. POAG, NVG, angle-recession glaucoma, lens dislocation, pseudoexfoliation syndrome, glaucoma associated with hemorrhage, congenital glaucoma, pigmentary glaucoma, glaucoma associated with inflammation, and glaucoma associated with aphakia or pseudophakia showed significant male predominance (assuming 50\% as an esti- mated sex ratio, $p<0.05$ by chi-square test). In contrast, AACG, CACG and glaucoma associated with corneal endothelial disorder showed female predominance (assuming $50 \%$ as an estimated sex ratio, $p<0.05$ by chi-square test) (Table 1). The presence of diabetes and hypertension varied widely (ranges, $0.0 \%$ to $47.4 \%$ and $0.0 \%$ to $37.8 \%$, respectively) in glaucoma subtypes. The presence of family history also showed a range of variation (range, $0.0 \%$ to $8.7 \%$ ) (Table 1). The ocular information for newly diag- 
nosed glaucoma patients is presented in Table 2. Mean IOP and vertical cup-to-disc ratio varied widely with glaucoma subtypes.

Of the newly diagnosed glaucoma patients, the 8 most frequently observed glaucoma subtypes accounted for $92.1 \%$ of the total. In the age distribution, CACG, AACG, NVG and glaucoma associated with aphakia or pseudophakia did not show significant differences ( $p>0.05$ by Student's $t$-tests); however, all other glaucoma subtypes showed significant differences ( $p<0.05$ by Student's $t$-tests). In terms of IOP, NVG and AACG did not show a significant difference ( $p>0.05$ by Student's $t$-test); however, all of the other glaucoma subtypes showed significant differences ( $p>0.05$ by Student's $t$-test). In cup-to-disc ratio, NTG, POAG, CACG, NVG, and glaucoma associated with aphakia or pseudophakia showed no significant difference ( $p=0.504$ by one-way analysis of variance); however, all other glaucoma subtypes showed a significant difference ( $p<0.05$ by Student's $t$-test).

In relation to the incidence rate of diabetes, NTG, OHT, CACG, glaucoma associated with inflammation, AACG, and glaucoma associated with aphakia or pseudophakia did not show any significant difference ( $p=0.102$ by chisquare test). However, POAG showed a significantly higher incidence rate of diabetes than did NTG or glaucoma associated with inflammation ( $p<0.05$ by chi-square tests). NVG showed a significantly higher incidence rate of diabetes than did any of the other glaucoma subtypes ( $p<$ 0.05 by chi-square test).

Regarding the incidence rate of systemic hypertension, glaucoma associated with inflammation showed a significantly lower incidence rate than did any of the other glaucoma subtypes ( $p<0.05$ by chi-square test); but NVG showed a significantly higher incidence rate than did any of the other glaucoma subtypes except for AACG $(p<0.05$ by chi-square test). NTG, POAG, NVG, CACG, and AACG did not show significant differences in terms of incidence rate of hypertension ( $p>0.05$ by chi-square test).

\section{Discussion}

In the current study, the sex distribution of AACG, subacute ACG, CACG, and glaucoma associated with corneal endothelial disorder exhibited significant female predominance. This finding is consistent with previous studies [19-
23]. In contrast, POAG, OHT, NVG, glaucoma associated with inflammation, glaucoma in aphakia or pseudophakia, angle-recession glaucoma, lens dislocation, and glaucoma associated with hemorrhage exhibited significant male predominance. We speculate that the male predominance among cases of angle-recession glaucoma, lens dislocation, and glaucoma associated with hemorrhage could be explained by the fact that these subtypes of glaucoma are usually associated with trauma. However, significant male predominance among POAG, OHT, NVG, glaucoma associated with inflammation, and glaucoma in aphakia or pseudophakia require further investigation. In the comparison of NTG with POAG, NTG showed a significantly higher female proportion and a lower age. Previous publications also reported a higher percentage of women in the NTG classification; thus our result was in line with these publications [24-26]. A higher proportion of lower age in NTG may be related to the recent observation that greater proportion of myopia is noted in the younger age group, and myopia is a risk factor for NTG [27]. This speculation requires further investigation. The reported association between diabetes and glaucoma remains controversial [2838]. According to official statistics from the Korean government (Korean National Health and Nutrition Examination Survey IV), the prevalence of systemic hypertension and diabetes in the general Korean population (age equal to or more than 30 years) were $24.9 \%$ and $9.5 \%$, respectively. The incidence rate of diabetes in glaucoma associated with inflammation was $5.2 \%$, which was lower than that of general Korean population. Meanwhile, the incidence rate of diabetes in POAG and glaucoma in aphakia or pseudophakia were found to be higher than that of the general Korean population (11.9\% and $14.1 \%$ vs. 9.5\%). However, we cannot directly compare these results because our results, based on outpatient clinic visits, may be prone to selection bias. The association between systemic hypertension and glaucoma, usually attributed to ocular perfusion pressure, is also debatable [39,40]. In this study, various subtypes of glaucoma showed a wide range of incidence rates in systemic hypertension $(0.0 \%$ to $31.5 \%)$. However, all glaucoma subtypes except NVG had a lower incidence rate of systemic hypertension than the general Korean population $(24.9 \%$ according to official Korean records-Korean National Health and Nutrition Examination Survey IV). Among the most frequently observed subtypes of glaucoma, CACG and AACG had a signifi- 
cantly higher incidence rate of systemic hypertension; however, this finding may be related to the observation that patients with CACG and AACG patients are relatively older.

In the Namil study, POAG with IOP $\leq 21 \mathrm{mmHg}(2.81 \%)$ was the most frequent subtype of glaucoma, followed by POAG with IOP $>21 \mathrm{mmHg}(0.78 \%)$, PACG $(0.65 \%)$, OHT $(0.59 \%)$, and secondary glaucoma (glaucoma associated with surgery, $0.26 \%$ ). In this study, based on a hospital-visiting population, POAG with IOP $\leq 21 \mathrm{mmHg}$ was the most frequently observed glaucoma subtype as in the Namil study. However, the proportion of POAG in this study was much greater than that of the Namil study. This can be explained by the fact that participants in this study were recruited from the clinic and thus may have had symptoms related to elevated IOP. However, because the Namil study was population-based, the study included asymptomatic patients. In the current study, the proportion of NVG and glaucoma associated with inflammation were much higher than that of Namil study. We speculate that this result reflects that more intractable types of glaucoma like NVG or glaucoma associated with inflammation are prone to manifest serious symptoms and signs. This results in more presentations to major glaucoma clinics.

This study had several limitations. First, this study included the patients who visited an ophthalmology outpatient clinic during the study period. Therefore, the subjects were enrolled in a hospital-based setting, which can be quite different from population-based studies. In addition, our study had a potential for referral bias: patients in tertiary medical centers were considerably different from the general population. The referral bias may have significantly affected the clinical characteristics and profiles of newly diagnosed glaucoma subtypes in this study. Also, analysis of the demographic and ocular factors that influence the referral of patients is necessary and should be performed in future analyses. Finally, the family history of various glaucoma types showed different profiles which can be a potentially interesting finding. Since family history was obtained by questionnaire, future study is warranted to reinforce our results.

In conclusion, a total of 198,671 patients were enrolled, $5,530(2.8 \%)$ of whom were newly diagnosed with glaucoma. NTG, POAG, OHT, CACG, NVG, glaucoma associated with inflammation, AACG, and glaucoma in aphakia or pseudophakia were the most frequently observed glauco- ma subtypes in the urban ophthalmology outpatient clinics included in this study. The incidence rate of diabetes in POAG and glaucoma in aphakia or pseudophakia were higher than that of the general Korean population. This is one of the largest studies investigating the profiles and clinical characteristics of glaucoma subtypes in urban clinics and thus can provide insight into the profile and clinical characteristics of those who visit urban ophthalmology outpatient clinics. Profiles of specific diseases in hospitals may reflect the corresponding prevalence of the general population. However, this prevalence may also be affected by many factors such as socioeconomic status and ethnicity, public health systems, and hospital access. Therefore, the findings in this study may not be representative of the general urban population in Korea. Moreover, as mentioned earlier, this was not a population-based study. Thus, the proportions of glaucoma reported herein should be interpreted with caution. Further population- based studies are warranted to determine the true prevalence of glaucoma subtypes in South Korean cities.

\section{Conflict of Interest}

No potential conflict of interest relevant to this article was reported.

\section{Acknowledgements}

\section{Collaborating Centers}

Myung Douk Ahn, MD (Catholic University), Young Joo Choi, MD (Siloam Eye Hospital), Chul Hong, MD (Dr. Hong's Eye Clinic), Sungpyo Hong, MD (Kyungbook National University), Young Jae Hong, MD (Yonsei University), Sung-Min Hyung, MD (Chungbuk National University), Hai Ryun Jung, MD (Korea University), Michael Scott Kook, MD (Asan Medical Center), Changwon Kee, MD (Sungkyunkwan University), Chan Yun Kim, MD (Yonsei University), Chang-sik Kim, MD (Chungnam National University), Dong Myung Kim, MD (Seoul National University), Ho Kyum Kim, MD (Il San Hospital), Jae-Woo Kim, MD (Catholic University of Daegu), Yong Yeon Kim, MD (Korea University), Chan Ju Lee, MD (Dr. C.J. Lee's Eye Clinic), Jong Hoon Lee, MD (Dankook University), Joo Hwa Lee, 
MD (Inje University), Kyoo Won Lee, MD (Cheil Eye Hospital), Kyung Wha Lee, MD (Hallym University), Sang Hyup Lee, MD (Pusan National University), Jung Il Moon, MD (Catholic University), Ki Ho Park, MD (Seoul National University), Sae Heun Rho, MD (Dong-A University), Gong Je Seong, MD (Yonsei University), Kun Jin Yang, MD (Chonnam National University), Dong Ho Youn, MD (Eulji University)

\section{Organizing and Data Monitoring Committee}

Ki Ho Park, MD, Youg Yeon Kim, MD, Changwon Kee, MD, Jung Il Moon, MD, and Chul Hong, MD

\section{Writing Committee}

Chan Kee Park, MD, Ki Ho Park, MD, Kyung Rim Sung, MD, Chang-sik Kim, MD, and Chul Hong, MD

\section{References}

1. Thylefors B, Negrel AD, Pararajasegaram R, Dadzie KY. Global data on blindness. Bull World Health Organ 1995;73:11521.

2. Quigley HA. Number of people with glaucoma worldwide. Br J Ophthalmol 1996;80:389-93.

3. Quigley HA, Broman AT. The number of people with glaucoma worldwide in 2010 and 2020. Br J Ophthalmol 2006;90:262-7.

4. Foster PJ, Oen FT, Machin D, et al. The prevalence of glaucoma in Chinese residents of Singapore: a cross-sectional population survey of the Tanjong Pagar district. Arch Ophthalmol 2000;118:1105-11.

5. Leske MC, Connell AM, Schachat AP, Hyman L. The Barbados Eye Study: prevalence of open angle glaucoma. Arch Ophthalmol 1994;112:821-9.

6. Bonomi L, Marchini G, Marraffa M, et al. Prevalence of glaucoma and intraocular pressure distribution in a defined population: the Egna-Neumarkt Study. Ophthalmology 1998;105:20915.

7. Buhrmann RR, Quigley HA, Barron Y, et al. Prevalence of glaucoma in a rural East African population. Invest Ophthalmol Vis Sci 2000;41:40-8.

8. Wensor MD, McCarty CA, Stanislavsky YL, et al. The prevalence of glaucoma in the Melbourne visual impairment project. Ophthalmology 1998;105:733-9.

9. Quigley HA, West SK, Rodriguez J, et al. The prevalence of glaucoma in a population-based study of Hispanic subjects: Proyecto VER. Arch Ophthalmol 2001;119:1819-26.

10. Rotchford AP, Johnson GJ. Glaucoma in Zulus: a population-based cross-sectional survey in a rural district in South Africa. Arch Ophthalmol 2002;120:471-8.

11. He M, Foster PJ, Ge J, et al. Prevalence and clinical characteristics of glaucoma in adult Chinese: a population-based study in Liwan District, Guangzhou. Invest Ophthalmol Vis Sci 2006;47:2782-8.

12. Tielsch JM, Sommer A, Katz J, et al. Racial variations in the prevalence of primary open-angle glaucoma: the Baltimore Eye Survey. JAMA 1991;266:369-74.

13. Foster PJ, Baasanhu J, Alsbirk PH, et al. Glaucoma in Mongolia: a population-based survey in Hövsgöl province, northern Mongolia. Arch Ophthalmol 1996;114:1235-41.

14. Shiose Y, Kitazawa Y, Tsukahara S, et al. Epidemiology of glaucoma in Japan: a nationwide glaucoma survey. Jpn $J$ Ophthalmol 1991;35:133-55.

15. Iwase A, Suzuki Y, Araie M, et al. The prevalence of primary open-angle glaucoma in Japanese: the Tajimi Study. Ophthalmology 2004;111:1641-8.

16. Xu L, Wang Y, Li Y, et al. Causes of blindness and visual impairment in urban and rural areas in Beijing: the Beijing Eye Study. Ophthalmology 2006;113:1134.

17. Wang Y, Xu L, Jonas JB. Prevalence and causes of visual field loss as determined by frequency doubling perimetry in urban and rural adult Chinese. Am J Ophthalmol 2006;141: 1078-86

18. Kim CS, Seong GJ, Lee NH, et al. Prevalence of primary open-angle glaucoma in central South Korea the Namil study. Ophthalmology 2011;118:1024-30.

19. Drance SM. Angle closure glaucoma among Canadian Eskimos. Can J Ophthalmol 1973;8:252-4.

20. Arkell SM, Lightman DA, Sommer A, et al. The prevalence of glaucoma among Eskimos of northwest Alaska. Arch Ophthalmol 1987;105:482-5.

21. Clemmesen V, Alsbirk PH. Primary angle-closure glaucoma (a.c.g.) in Greenland. Acta Ophthalmol (Copenh) 1971;49:4758.

22. Shields MB, Campbell DG, Simmons RJ. The essential iris atrophies. Am J Ophthalmol 1978;85:749-59.

23. Wilson MC, Shields MB. A comparison of the clinical variations of the iridocorneal endothelial syndrome. Arch Ophthalmol 1989;107:1465-8.

24. Sugiyama K, Tomita G, Kitazawa Y, et al. The associations of optic disc hemorrhage with retinal nerve fiber layer de- 
fect and peripapillary atrophy in normal-tension glaucoma. Ophthalmology 1997;104:1926-33.

25. Lee JY, Sung KR, Lee JY. Comparison of the progression of high- and low-tension glaucoma as determined by two different criteria. Korean J Ophthalmol 2016;30:40-7.

26. Sung KR, Cho JW, Lee S, et al. Characteristics of visual field progression in medically treated normal-tension glaucoma patients with unstable ocular perfusion pressure. Invest Ophthalmol Vis Sci 2011;52:737-43.

27. Shim SH, Sung KR, Kim JM, et al. The prevalence of open-angle glaucoma by age in myopia: the Korea national health and nutrition examination survey. Curr Eye Res 2017;42:65-71.

28. European Glaucoma Prevention Study (EGPS) Group, Miglior S, Pfeiffer N, et al. Predictive factors for open-angle glaucoma among patients with ocular hypertension in the European Glaucoma Prevention Study. Ophthalmology 2007;114:3-9.

29. Dielemans I, de Jong PT, Stolk R, et al. Primary open-angle glaucoma, intraocular pressure, and diabetes mellitus in the general elderly population: the Rotterdam Study. Ophthalmology 1996;103:1271-5.

30. Kahn HA, Leibowitz HM, Ganley JP, et al. The Framingham Eye Study. II. Association of ophthalmic pathology with single variables previously measured in the Framingham Heart Study. Am J Epidemiol 1977;106:33-41.

31. Klein BE, Klein R, Moss SE. Intraocular pressure in diabetic persons. Ophthalmology 1984;91:1356-60.

32. Klein BE, Klein R, Jensen SC. Open-angle glaucoma and older-onset diabetes: the Beaver Dam Eye Study. Ophthal- mology 1994;101:1173-7.

33. Gordon MO, Beiser JA, Brandt JD, et al. The ocular hypertension treatment study: baseline factors that predict the onset of primary open-angle glaucoma. Arch Ophthalmol 2002;120:714-20.

34. Leibowitz HM, Krueger DE, Maunder LR, et al. The Framingham Eye Study monograph: An ophthalmological and epidemiological study of cataract, glaucoma, diabetic retinopathy, macular degeneration, and visual acuity in a general population of 2631 adults, 1973-1975. Surv Ophthalmol 1980;24:335-610.

35. Leske MC, Connell AM, Wu SY, et al. Risk factors for openangle glaucoma: the Barbados Eye Study. Arch Ophthalmol 1995;113:918-24.

36. Mitchell P, Smith W, Chey T, Healey PR. Open-angle glaucoma and diabetes: the Blue Mountains eye study, Australia. Ophthalmology 1997;104:712-8.

37. Pasquale LR, Kang JH, Manson JE, et al. Prospective study of type 2 diabetes mellitus and risk of primary open-angle glaucoma in women. Ophthalmology 2006;113:1081-6.

38. Tielsch JM, Katz J, Quigley HA, et al. Diabetes, intraocular pressure, and primary open-angle glaucoma in the Baltimore Eye Survey. Ophthalmology 1995;102:48-53.

39. Tielsch JM, Katz J, Sommer A, et al. Hypertension, perfusion pressure, and primary open-angle glaucoma: a population-based assessment. Arch Ophthalmol 1995;113:216-21.

40. Bonomi L, Marchini G, Marraffa M, et al. Vascular risk factors for primary open angle glaucoma: the Egna-Neumarkt Study. Ophthalmology 2000;107:1287-93. 